\title{
Synthesis of Component and Connector Models from Crosscutting Structural Views (extended abstract)
}

\author{
Shahar Maoz \\ School of Computer Science \\ Tel Aviv University, Israel
}

\author{
Jan Oliver Ringert, Bernhard Rumpe \\ Software Engineering \\ RWTH Aachen University, Germany
}

\begin{abstract}
This extended abstract reports on [MRR13]. We presented component and connector $(\mathrm{C} \& \mathrm{C})$ views, which specify structural properties of component and connector models in an expressive and intuitive way. $\mathrm{C} \& \mathrm{C}$ views provide means to abstract away direct hierarchy, direct connectivity, port names and types, and thus can crosscut the traditional boundaries of the implementation-oriented hierarchical decomposition of systems and sub-systems, and reflect the partial knowledge available to different stakeholders involved in a system's design.

As a primary application for $\mathrm{C} \& \mathrm{C}$ views we investigated the synthesis problem: given a $\mathrm{C} \& \mathrm{C}$ views specification, consisting of mandatory, alternative, and negative views, construct a concrete satisfying $\mathrm{C} \& \mathrm{C}$ model, if one exists. We showed that the problem is NP-hard and solved it, in a bounded scope, using a reduction to SAT, via Alloy. We further extended the basic problem with support for library components, specification patterns, and architectural styles. The result of synthesis can be used for further exploration, simulation, and refinement of the $\mathrm{C} \& \mathrm{C}$ model or, as the complete, final model itself, for direct code generation.

A prototype tool and an evaluation over four example systems with multiple specifications show promising results and suggest interesting future research directions towards a comprehensive development environment for the structure of component and connector designs.
\end{abstract}

Component and connector $(\mathrm{C} \& \mathrm{C})$ models are used in many application domains, from cyber-physical and embedded systems to web services to enterprise applications. The structure of a $\mathrm{C} \& \mathrm{C}$ model consists of components at different containment levels, their typed input and output ports, and the connectors between them.

A system's C\&C model is typically complex; it is not designed by a single engineer and is not completely described in a single document. Thus, we considered a setup where many different, incomplete, relatively small fragments of the model are provided by architects responsible for subsystems, for the implementation of specific features, use cases, or functionality, which crosscut the boundaries of components. Moreover, teams may have several, alternative solutions that address the same concern, and some knowledge about designs that must not be used. To move forward in the development process and enable implementation, these partial models and the intentions behind them should be integrated and then realized into a single, complete design. However, such an integration is a complex and challenging task.

In [MRR13] we presented component and connector views, which specify structural properties of component and connector models in an expressive and intuitive way. C\&C views 
provide means to abstract away direct hierarchy, direct connectivity, port names and types. Specifically, C\&C views may not contain all components and connectors (typically a small subset related only to a specific use case or set of functions or features). They may contain (abstract) connectors between components at different, non-consecutive containment levels, and they may provide incomplete typing information, that is, components' ports may be un-typed. While the standard structural abstraction and specification mechanisms for $\mathrm{C} \& \mathrm{C}$ models rely on the traditional, implementation-oriented hierarchical decomposition of systems to sub-systems, $\mathrm{C} \& \mathrm{C}$ views allow one to specify properties that crosscut the boundaries of sub-systems. This makes them especially suitable to reflect the partial knowledge available to different stakeholders involved in a system's design.

As a primary application for $\mathrm{C} \& \mathrm{C}$ views we investigated the synthesis problem: given mandatory, alternative, and negative views, construct a concrete satisfying $\mathrm{C} \& \mathrm{C}$ model, if one exists. We have shown that the synthesis problem for $\mathrm{C} \& \mathrm{C}$ views specifications is NPhard and solved it, in a bounded scope, using a reduction to Alloy [Jac06]. The input for the synthesis is a $\mathrm{C} \& \mathrm{C}$ views specification. Its output is a single $\mathrm{C} \& \mathrm{C}$ model that satisfies the specification and is complete, to allow implementation. When no solution exists (within a bounded scope), the technique reports that the input specification is unsatisfiable.

As a concrete language for $\mathrm{C} \& \mathrm{C}$ models we used MontiArc [HRR12], a textual ADL developed using MontiCore [KRV10], with support for direct Java code generation (including interfaces, factories, etc.). The $\mathrm{C} \& \mathrm{C}$ views are defined as an extension to general $\mathrm{C} \& \mathrm{C}$ models. The concrete syntax used in our implementation is an extension of MontiArc.

To further increase the usefulness of $\mathrm{C} \& \mathrm{C}$ views synthesis in practice, we extended the basic synthesis problem with support for three advanced features. First, support for integration with pre-defined or library components. Second, support for several high-level specification patterns. Third, support for synthesis subject to several architectural styles.

We implemented $\mathrm{C} \& \mathrm{C}$ views synthesis and evaluated it by applying it to four example systems. The implementation and example specifications are available from [www].

\section{References}

[HRR12] Arne Haber, Jan Oliver Ringert, and Bernard Rumpe. MontiArc - Architectural Modeling of Interactive Distributed and Cyber-Physical Systems. Technical Report AIB-2012-03, RWTH Aachen, february 2012.

[Jac06] Daniel Jackson. Software Abstractions: Logic, Language, and Analysis. MIT Press, 2006.

[KRV10] Holger Krahn, Bernhard Rumpe, and Steven Völkel. MontiCore: a framework for compositional development of domain specific languages. STTT, 12(5):353-372, 2010.

[MRR13] Shahar Maoz, Jan Oliver Ringert, and Bernhard Rumpe. Synthesis of Component and Connector Models from Crosscutting Structural Views. In Bertrand Meyer, Luciano Baresi, and Mira Mezini, editors, ESEC/SIGSOFT FSE, pages 444-454. ACM, 2013.

[www] C\&C Views Synthesis Evaluation Materials. http://www.se-rwth.de/ materials/cncviews/. 